\title{
Intracardiac Acoustic Radiation Force Impulse (ARFI) and Shear Wave Imaging in Pigs With Focal Infarctions
}

\author{
Peter Hollender, Student Member, IEEE, David Bradway, Student Member, IEEE, \\ Patrick Wolf, Member, IEEE, Robi Goswami, and Gregg Trahey, Member, IEEE
}

\begin{abstract}
Four pigs, three with focal infarctions in the apical intraventricular septum (IVS) and/or left ventricular free wall (LVFW), were imaged with an intracardiac echocardiography (ICE) transducer. Custom beam sequences were used to excite the myocardium with focused acoustic radiation force (ARF) impulses and image the subsequent tissue response. Tissue displacement in response to the ARF excitation was calculated with a phase-based estimator, and transverse wave magnitude and velocity were each estimated at every depth. The excitation sequence was repeated rapidly, either in the same location to generate $40 \mathrm{~Hz} \mathrm{M}$-modes at a single steering angle, or with a modulated steering angle to synthesize 2-D displacement magnitude and shear wave velocity images at 17 points in the cardiac cycle. Both types of images were acquired from various views in the right and left ventricles, in and out of infarcted regions. In all animals, acoustic radiation force impulse (ARFI) and shear wave elasticity imaging (SWEI) estimates indicated diastolic relaxation and systolic contraction in noninfarcted tissues. The M-mode sequences showed high beat-to-beat spatio-temporal repeatability of the measurements for each imaging plane. In views of noninfarcted tissue in the diseased animals, no significant elastic remodeling was indicated when compared with the control. Where available, views of infarcted tissue were compared with similar views from the control animal. In views of the LVFW, the infarcted tissue presented as stiff and non-contractile compared with the control. In a view of the IVS, no significant difference was seen between infarcted and healthy tissue, whereas in another view, a heterogeneous infarction was seen to be presenting itself as non-contractile in systole.
\end{abstract}

\section{INTRODUCTION}

\section{A. Myocardial Infarction}

Bogen et al. [1] notes that myocardial infarction is characterized by a localized increase in myocardial stiffness and decreased contractility that evolves over weeks after the infarction event. Increased wall stresses in the rest of the ventricle cause it to grow and thin, increasing dysfunc-

Manuscript received December 10, 2012; accepted April 28, 2013 This research was supported by the National Institutes of Health (NIH) through Medical Imaging Training Grant EB001040, NIH R37HL096023 and NIH R01EB01248.

P. Hollender, D. Bradway, P. Wolf, and G. Trahey are with the Department of Biomedical Engineering, Duke University, Durham, NC (email: peter.hollender@duke.edu).

R. Goswami is with the Department of Cardiology, Duke University Medical Center, Durham, NC.

G. Trahey is with the Department of Radiology, Duke University, Durham, NC

DOI http://dx.doi.org/10.1109/TUFFC.2013.2749 tion and the subsequent risk of heart failure. The infarcted tissue is replaced by collagen, forming a stiff, fibrous scar [2], [3]. Concurrent with scar formation, the ventricles may remodel to compensate for the thinned, scarred segments being unable to support wall stresses. Remodeling is a change in the size, shape, and function of the ventricle, and is often a precursor to eventual heart failure.

Infarctions can be imaged with several modalities, including contrast-enhanced magnetic resonance imaging (MRI) [4]-[7], positron emission tomography (PET) [8], computed tomography (CT) [9], and most commonly, echocardiography [10]-[12]. Echocardiography is used to diagnose wall motion abnormalities as an indicator for infarction, but does not distinguish dead myocardium from "stunned" [13]-[15] or otherwise dysfunctional myocardium. A means to distinguish necrotic, stunned, hibernating, edemic, and healthy regions of tissue in the emergency room could greatly improve diagnoses and management [16], [17].

\section{B. Acoustic Radiation Force Impulse and Shear Wave Elasticity Imaging}

The motional response of tissue to external forces can be used to infer information about the tissue's mechanical or elastic properties. Acoustic radiation force impulse (ARFI) imaging uses focused, 10- to 100- $\mu$ s ultrasonic pulses to locally transfer momentum into the tissue via absorption. The tissue transiently displaces away from the transducer around the focus of the excitation, and is concurrently imaged with high-frame-rate ultrasound. The spatial distribution of induced displacement magnitude is a function of not only the excitation beam profile, but also of the local elasticity of the excited tissue. Another way to characterize elasticity is to image the propagation velocity of transverse (shear) waves through a medium. Under a linear, semi-infinite, purely-elastic assumption, the shear wave velocity is related to the shear modulus by $c_{\mathrm{T}}=$ $\sqrt{\mu / \rho}$, where $c_{\mathrm{T}}$ is the velocity in meters per second, $\mu$ is the shear modulus in kilopascals, and $\rho$ is the density, in grams per cubic centimeter. This is known as shear wave elasticity imaging (SWEI), and acoustic radiation force (ARF) excitations provide a convenient way to generate shear waves within the ultrasonic field of view (FOV).

Cardiac elasticity imaging with these ultrasonic techniques and others has been an area of increasing interest for its potential in directly imaging the mechanical com- 
pliance of dysfunctional, infarcted, or fibrotic regions of myocardial tissue [18]-[24]. These studies have all imaged a transient mechanical response with ultrasound to characterize myocardial stiffness. Kanai imaged the transient Lamb wave generated in the IVS in response to aortic valve closure, but the other work has required external excitation of the myocardium, either mechanically or with ARF. To generate a highly localized excitation, the animals' chests were opened and the excitation transducer was coupled directly to the myocardium.

Intracardiac echocardiography (ICE) holds promise for obtaining the proximity to the tissue needed to generate and track tissue response to ARF excitations. ICE is commonly used for function assessment and therapy guidance [25]-[29], and has already been shown to be feasible for cardiac ARFI imaging [30], [31]. Because the imaging transducer is located so close to the myocardium, less ultrasonic energy is needed to excite the tissue, and the response can be imaged without the clutter associated with imaging across the chest wall. This work uses an intracardiac implementation to explore the possibility of using SWEI and ARFI imaging to distinguish healthy myocardium from infarcted myocardium. Although a transthoracic means to image cardiac elasticity with ultrasound would have the greatest clinical potential for diagnosing acute myocardial infarction (AMI), this implementation may benefit patients in procedures where ICE is already called for and the location of infarcted myocardium is of interest (transcatheter ablation or pacemaker lead placement, for example). Moreover, this work aims to explore the elastic appearance and behavior of infarctions in a closed-chest animal model.

\section{MethodS}

\section{A. Experimental Setup}

All data presented were acquired with 10-French ICE catheters, either the Acuson AcuNav (Siemens Medical Systems, Mountain View, CA) or the SoundStar (Biosense Webster Inc., Diamond Bar, CA), running on a Siemens S2000 scanner. Both transducers use the same 64-element, $7.25 \mathrm{MHz}$ linear phased array, with a $7-\mathrm{mm}$ aperture in azimuth, but the SoundStar has an additional magnetic positioning sensor in its tip. Demodulated in-phase and quadrature (IQ) ultrasound data from custom pulse sequences were recorded with matched ECG data from eight porcine subjects in compliance with protocols from Duke University's Institutional Animal Care and Use Committee (IACUC). One of the pigs was used as a control, and the other seven were subjected to ischemia through a series of foam embolizations to the left anterior descending (LAD) coronary artery, designed to mimic chronic ischemia in the apical IVS and lead to heart failure, but which ultimately created a pathology more like AMI. Additionally, in all but one of the initial injections, foam was unexpectedly also delivered to the left circumflex (LCX) coronary, resulting in infarction of the LVFW as well. Unfortunately, four of the animals did not survive to the first follow-up study. The four remaining animals (three embolized and one control) comprise the cohort for the data presented here.

The ICE catheter was either fed through the right atrium into the right ventricle (RV), or inserted retrograde across the aortic valve into the left ventricle $(\mathrm{LV})$. The animals were each imaged four times: once before the embolization, and three times following, over a time period of approximately $150 \mathrm{~d}$. The first follow-up imaging session was at $40 \pm 12 \mathrm{~d}$ after embolization, the second at $53 \pm$ $11 \mathrm{~d}$, and the final study at $130 \pm 24 \mathrm{~d}$. The catheter was placed in the LV only during the final imaging study for safety purposes, after which the animals were sacrificed and their hearts sent for contrast-enhanced MRI imaging and subsequent histology. Imaging planes were selected to view the ventricular free wall, either from the RV outflow tract or near the apex, as well as the IVS, under the condition that the tissue was at most $20 \mathrm{~mm}$ from the transducer face. In each view, up to three M-mode and one 2-D data sets were collected. In each imaging session, at least one view of the IVS and one view of the right ventricular free wall (RVFW) were obtained. Where independent views of the IVS or RVFW could be obtained, additional views of the same wall were imaged, especially when the presence of infarction was suspected. A total of 196 data sets were collected across the 16 imaging sessions.

\section{B. Custom Beam Sequences}

The impulsive excitation was generated with a 6.15$\mathrm{MHz} 400$-cycle $(65 \mu \mathrm{s})$ pulse, using a single focus selected to maximally intersect the myocardium throughout the cardiac cycle. Available foci were every $2.5 \mathrm{~mm}$ between 7.5 and $20 \mathrm{~mm}$. Previous intensity measurements and thermal finite element modeling [32] (data not shown) indicated that for similar pulse sequences $(15 \mathrm{~mm}$ focus and $6 \mathrm{MHz}$ center frequency), the mechanical index (MI) is less than 1.63 , the $I_{\mathrm{SPPA}}$ is less than $2000 \mathrm{~W} / \mathrm{cm}^{2}$ (derated at $\alpha=0.3$, and tissue heating at the focus is low, $1.01^{\circ} \mathrm{C}$ over $4 \mathrm{~s}$ with 80 excitations per second. The $I_{\mathrm{SPTA}}$ will thus be as high as $10 \mathrm{~W} / \mathrm{cm}^{2}$ during transmission of ARF pulses at 80 times per second. The beams were repeated and steered in two different tracking schema, to make the two types of images. A summary of the parameters is found in Table I.

1) M-Mode ARFI-SWEI Sequences: To generate high temporal sampling of the cardiac dynamics, the excitation beam was held in the same location and rapidly repeated to generate M-modes of shear wave velocity and displacement magnitude. The tissue response was measured between $0^{\circ}$ and $15^{\circ}$ to the right of the center of the B-mode field of view as the superposition of two identically-generated responses, each imaged at different lateral locations. A diagram of the tracking configuration is shown in Fig. 1(a). Four parallel tracking beams were simultaneously 
TABle i. Parameters for Imaging Sequences.

\begin{tabular}{lc}
\hline Common parameters & \\
Center frequency & $6.15 \mathrm{MHz}$ \\
PRF & $9.12 \mathrm{kHz}$ \\
Excitation pulse duration & $64 \mathrm{~s}$ \\
Excitation focus range & 7.15 to $20 \mathrm{~mm}$ \\
Tracking pulse bandwidth & $50 \%$ \\
Tracking transmit focus & $400 \mathrm{~mm}$ \\
Imaging depth & $40 \mathrm{~mm}$ \\
B-mode parameters & \\
Field of view & $90^{\circ}$ \\
Beam spacing & $0.65^{\circ}$ \\
M-mode ARFI-SWEI parameters & $0^{\circ}$ \\
Excitation steering angle & $15^{\circ}$ right of center \\
Tracking ROI & 16 \\
Tracking lines & $5 \mathrm{~ms}$ \\
Tracking duration & 2 \\
Excitations per ensemble & $40 \mathrm{~Hz}$ \\
Ensemble PRF & 160 \\
Number of ensembles & \\
Synthesized 2-D ARFI-SWEI parameters & $-22.5^{\circ}$ to $+22.5^{\circ}$ \\
Excitation steering angles & $2.7^{\circ}$ \\
Excitation angular spacing & $3 \mathrm{~ms}$ \\
Tracking duration & $16^{\circ}$ right of each excitation \\
Tracking ROI & 1 \\
Tracking lines per excitation (ARFI) & 3 \\
Tracking lines per excitation (SWEI) & 17 \\
Number of synthesized images & \\
\hline & \\
\hline
\end{tabular}

recorded at a maximum depth of $40 \mathrm{~mm}$, repeated at $9.12 \mathrm{kHz}$ for $5 \mathrm{~ms}$ following each excitation. The receive beams directions were rapidly modulated during tracking, such that each receive beam alternately sampled two locations at $4.56 \mathrm{kHz}$ each, for a total of 8 tracked angles per excitation. The tracking locations were changed on the second excitation for a total of 16 tracking lines across the two excitations. The tracking transmit beams had a $6.15 \mathrm{MHz}$ center frequency, and were focused at $400 \mathrm{~mm}$ to distribute transmit energy across the dynamic-receive focused-receive beam locations. The entire excitationtracking ensemble was repeated 40 times per second for $4 \mathrm{~s}$, with a $90^{\circ}$ field-of-view B-mode image acquired between each ensemble.

2) Synthesized 2-D ARFI-SWEI Sequences: An ECGgated multi-beat synthesis method was used to generate sequences of 2-D ARFI and SWEI images that image multiple locations throughout the cardiac cycle. Because the excitation-response ensemble takes at least $5 \mathrm{~ms}$ per

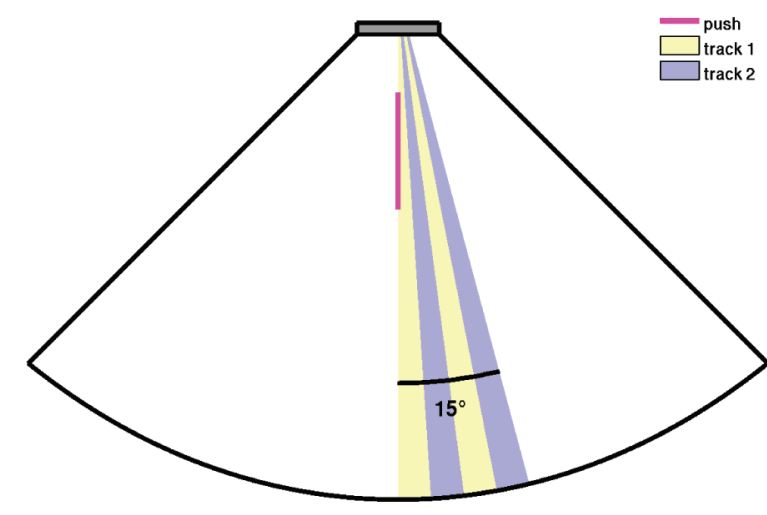

(a)

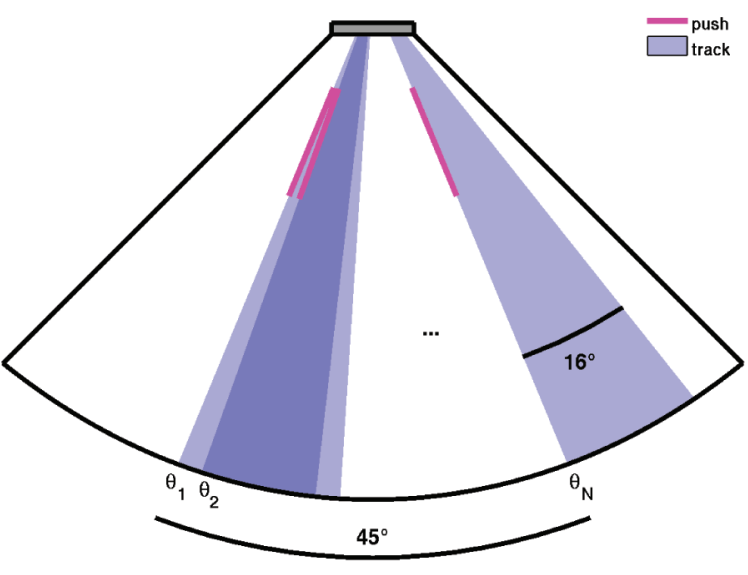

(b)

Fig. 1. (a) Locations of the M-mode ARFI-SWEI excitation and tracking regions. Four closely-spaced parallel tracking lines are rapidly steered between the two yellow tracking regions following the first excitation, and between the two blue tracking regions after the second excitation, for a total of 16 tracking lines. (b) Locations of the synthesized 2-D ARFI-SWEI image sequence acquisition ROIs. Four parallel tracking lines cover $16^{\circ}$ to the right of each of the $N$ excitation locations. In this work, $N=17$ and the excitations are spaced $2.66^{\circ}$ apart. 


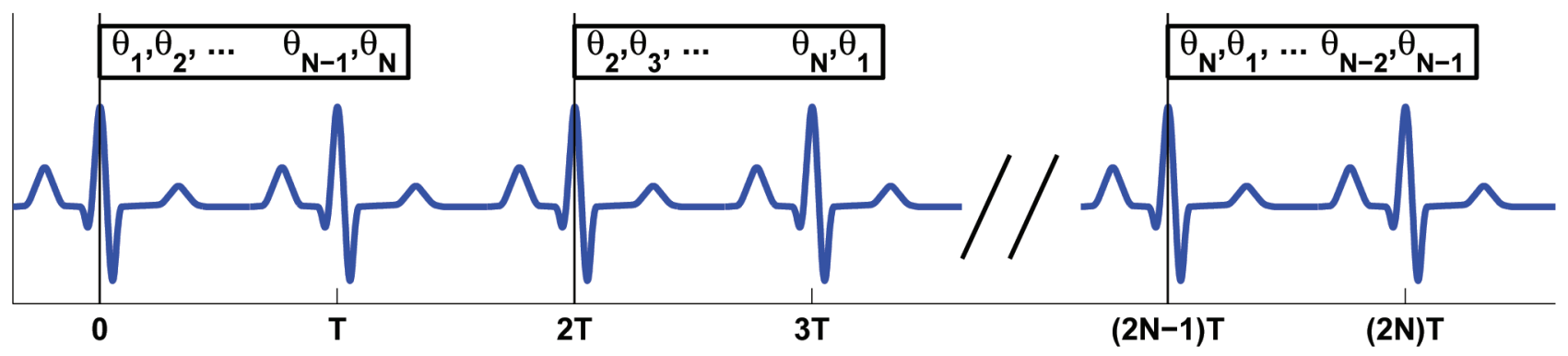

Fig. 2. The ECG-triggered acquisition scheme used to synthesize 2-D ARFI and SWEI image sequences. Each box represents an acquisition, indicating the order in which the scan lines are excited and tracked. The width of each box is the total time to acquire all $N$ scan lines in each acquisition. $T$ is the RR-Interval, and the vertical black lines represent ECG trigger signals. The work in this paper uses $N=17$.

line, each ARFI or SWEI image takes much longer to acquire than a B-mode image; in moving or changing imaging targets, this introduces artifacts associated with the time delays between imaging each angle. To address this, custom sequences were written that change the order in which the angles are imaged on each successive beat. A diagram is shown in Fig. 2. Over several beats, each angle in the field of view gets imaged at each delay in the cardiac cycle. There are several ways to configure the modulation of angles and delays with ECG triggering, but we selected a method that uses 17 excitations per image, and synthesizes 17 images over a configurable time selected to cover approximately one beat to stay within the memory restrictions of our system. On the first beat, the excitation steering angle progressed from left to right in $2.66^{\circ}$ increments at intervals of $1 / 17$ th of the $\mathrm{R}-\mathrm{R}$ interval, starting on the first QRS complex at the leftmost beam (beam 1) and ending approximately on the following QRS complex with the rightmost beam (beam 17). B-modes were acquired between each ensemble, and the delay between ensembles was set so that the last beam was acquired approximately a full beat after the first. On the second beat, the ensembles progressed from left to right again, but started at beam 2 on the QRS complex, and then acquire beams 3 through 17 , followed finally by beam 1 . The pattern continued as the third beat started with beam 3 , the fourth with beam 4 , and so on and so forth to the final acquisition, which excited first beam 17 on the QRS, followed by beams 1 through 16 over the final beat. The entire sequence actually took 34 heartbeats to acquire (rather than the minimum of 17), because the ECG trigger could not be configured to fire immediately following each set of 17 ensembles, requiring an extra beat between each acquisition set to reset. To track the on-axis (ARFI) and off-axis propagation (SWEI) from each excitation, four receive beams were used, one aligned with the excitation, and the other three spanning $16^{\circ}$ to the right of the acquisition, as shown in Fig. 1(b). To reduce respiration motion artifacts in the synthesized image sequences, respiration was temporarily held during acquisition. More lateral locations or time steps could not be synthesized with this setup, because our 17-time, 17-push-location image sequences generated the largest files that could be saved by our scanner (200 MB).

\section{Shear Wave Speed Estimation}

A phase-shift based displacement estimator [33] was used to calculate the ARF-induced axial displacements, using a 1.5- $\lambda$ kernel, progressively calculating the displacements between each successive pair of frames in the ensemble. To calculate the total displacement from the pairwise estimates, the stepwise displacement estimates were integrated. Once the displacements had been calculated, a third-order polynomial motion filter was used to remove the bulk axial motion of the tissue from the ARF-induced displacements [34]. For each ensemble in the M-mode data, the temporal displacement estimate profiles were linearly interpolated up to $9.12 \mathrm{kHz}$ to fill in the missing time steps caused by the steering angle modulation. These displacement data, when tracked at the same steering angle as the excitation, are referred to as ARFI displacements; the rest of the data are used for velocity estimation.

At each depth and steering angle, the time to peak slope, or arrival time, was estimated using a sub-sample peak estimator that fit a quadratic polynomial to the peak rise in displacement and its temporally-adjacent estimates. Spatio-temporal samples corresponding to shear wave speeds less than $0.5 \mathrm{~m} / \mathrm{s}$ or greater than $8 \mathrm{~m} / \mathrm{s}$ were not considered when selecting the peak value. Simple linear regression was performed on all of the arrival times at each depth to obtain an estimate of the transverse wave velocity [35]. These velocity estimates are also referred to as SWEI [36] and shear wave speed estimates. The estimation process was repeated independently for each repeated excitation ensemble. The M-mode sequences formed 470 $\times 160$ pixel M-mode images through depth and across multiple heartbeats, to which a $5 \times 3$ pixel median filter was applied to remove outliers. For the synthesized 2-D images, the ARFI and SWEI estimates from each response were sorted to form sets of data, where each set contained all of the responses for a given ECG trigger delay, one at each lateral steering angle. For all of the ensembles acquired with the same delay, each shear wave speed estimate was assigned to the $15^{\circ}$ region of interest (ROI) from which it was calculated and overlapping estimates for each lateral line were averaged together, weighted by the magnitude of their complex cross-correlation coefficients. The 
ARFI data did not overlap, so they were assigned directly to their lateral locations.

\section{Multi-Beat Synthesis}

To compile the data acquired across multiple heartbeats, a technique called multi-beat synthesis [37] was used to register estimates from many frames onto a single heartbeat. Using matched ECG data, each displacement and velocity estimate was registered to the preceding QRS complex, placing all estimates on a single beat. For the 2-D image sequences, this resulted in a single estimate at each combination of steering angle and cardiac phase, making a series of seventeen $470 \times 17$ pixel (pre-scanconversion) ARFI images. SWEI images were similarly formed, but had an additional $16^{\circ}$ in their field of view because of the extent of the rightmost tracking kernel [Fig. 2 (b)], which resulted in $470 \times 23$ pixel images. For the M-mode sequences, the 160 temporal samples cover all phases of the cardiac cycle for the center steering angle. Forty evenly spaced time bins were used to subdivide one heart cycle, with each of the 160 estimates placed into the nearest bin. The estimates within each bin were then averaged, weighted by their correlation coefficients, to form two $470 \times 40$ pixel M-modes, one for ARFI displacement and one for shear wave speed.

\section{E. Model Fitting}

To form a more quantitative comparison among Mmode images, 10 averaged profiles of displacement and velocity were computed from each synthesized M-mode. The borders of the tissue were drawn by hand using the center line of the B-modes for guidance, and the profiles averaged between deciles spaced between the axial boundaries so that each profile followed the same piece of axially-moving myocardium. Each of the 10 ARFI or SWEI profiles was then fit to a simple, six-parameter piecewise-linear model. The parameters were diastolic value, systolic value, enddiastole time, diastolic-systolic transition time, systolic duration, and systolic-diastolic transition time. A shear wave speed temporal profile for a single location and its corresponding model are shown in Fig. 3. During diastole and systole, the model is constant, and during the transition times, the model is linear. MATLAB's fit function (The MathWorks Inc., Natick, MA) was used, implementing a constrained, robust, nonlinear least squares Levenberg-Marquardt algorithm to do the fitting. The model is simple, but allows a numeric comparison between large amounts of data contained in each M-mode. The parameters of interest reported in this work are the diastolic and systolic values.

\section{RESUlts}

Over all animals and all imaging sessions, $154 \mathrm{M}$-mode images and 42 2-D image sequences were acquired in vari-

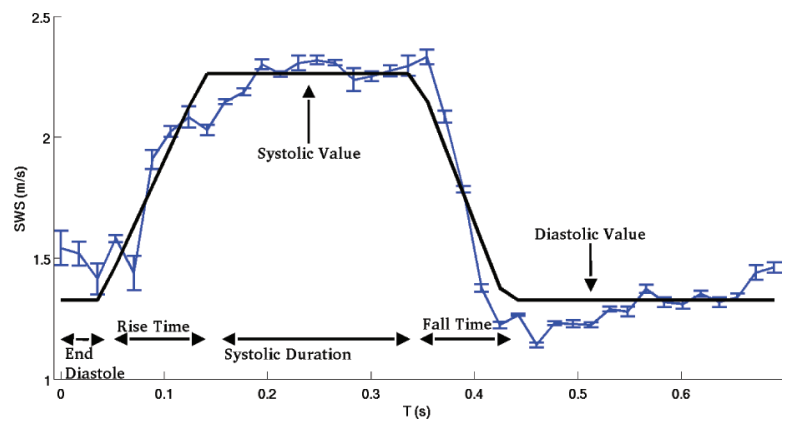

Fig. 3. Single-depth shear wave speed temporal profile with the simple model overlaid. The model parameters are fit to the means at each time step.

ous views of the left and right ventricle. The results presented here are a subset of the views for which lateral tissue motion and the imaging angle were comparable.

\section{A. Left Ventricular Free Wall Infarction}

Fig. 4 shows a single frame of the B-mode clips acquired in the LVFW of one of the infarcted pigs on the left and the control pig on the right. The left image shows a large focal infarct in the LVFW; the right image is nearly the same view, but shows no signs of damage. ARFI and SWEI estimates will be compared in the data acquired from these views.

Fig. 5 shows the M-modes of displacement magnitude and shear wave speed for the control pig's LVFW over six beats. The axial and temporal trends in shear wave speed and velocity are highly repeatable from beat to beat and correspond with the associated ECG. It is also of note that depth variation is seen in the SWEI image but not in the ARFI image.

Fig. 6 shows six frames from the multi-beat synthesis 2-D SWEI and ARFI image sequence for the same view shown in Fig. 5. A similar trend of elevated shear velocities and suppressed displacements are seen during systole, compared with diastole. Dimmed pixels, which show the underlying B-mode, correspond to low cross-correlation coefficients or regions outside of the myocardium.
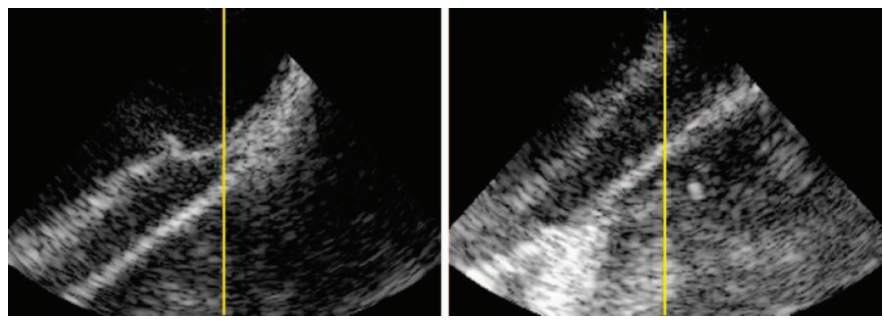

Fig. 4. B-mode frames of the left ventricular free wall (LVFW). The left image is taken from one of the infarcted pigs $126 \mathrm{~d}$ after infarction, and shows a large focal infarct in the frame. The right image, from the control (146 d after its sham procedure), shows the same view, but without any infarction. The yellow line indicates the steering angle of the ARF excitation for the M-mode sequences. 

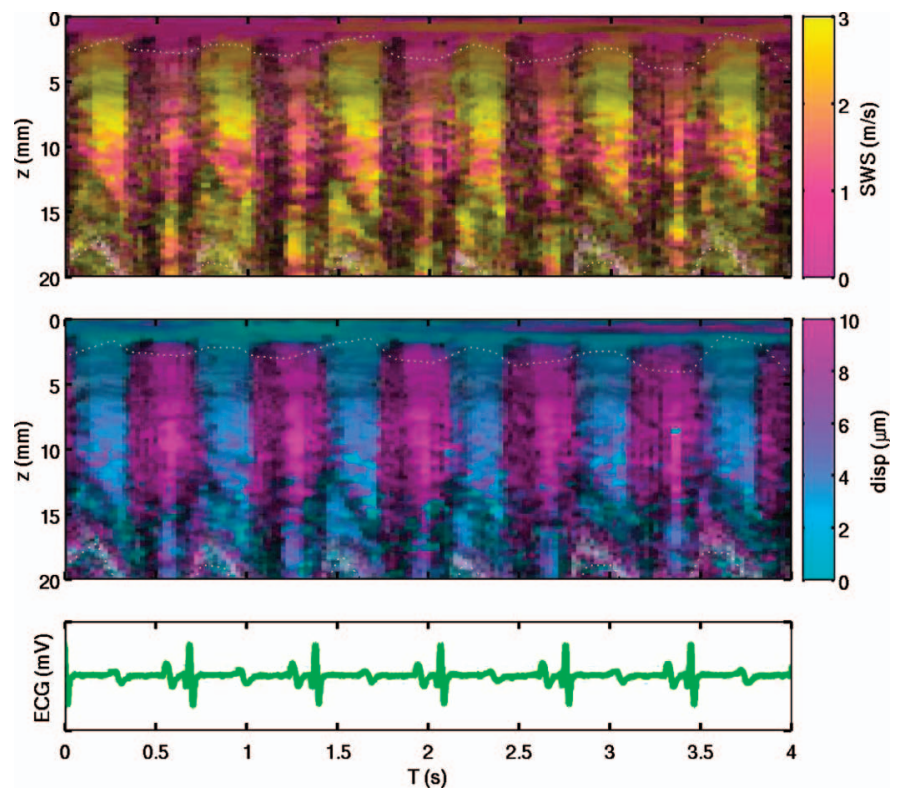

Fig. 5. (top row) SWEI and (middle row) ARFI M-modes of the left ventricular free wall (LVFW) from the control animal, with (bottom row) the corresponding ECG. Data were taken $146 \mathrm{~d}$ after the first imaging date. High shear wave speeds and low displacements (yellow and cyan, respectively) correspond to stiff myocardium in systole, and slow speeds and high displacements (magenta) correspond to compliant myocardium. Desaturated pixels correspond to points outside of the depth of field and those with low complex cross-correlation coefficients. In each of the six beats, the axial and temporal trends in both ARFI and SWEI measurements are highly repeatable.
Fig. 7 shows the SWEI and ARFI M-modes for the two views in Fig. 4 after multibeat synthesis, along with the associated ECG and (uncalibrated) LV pressure curve. The diastolic speeds appear elevated in the infarction compared with the control, and the displacements are correspondingly lower in the infarction. A small amount of relaxation is seen in the infarction from 350 to $500 \mathrm{~ms}$.

Box plots are shown in Fig. 8 for the model parameters fit to the data in Fig. 7 and the rest of the data sets from those views. Statistically significant difference in the means at the $5 \%$ level is indicated by the shear wave speeds for all Infarct-Healthy pairs except Infarct 2 and Healthy 3. In the ARFI displacement data, statistical significance is only indicated between Infarct 2 and Healthy 1 and Infarct 2 and Healthy 2.

Fig. 9 shows the same frames from the 2-D sequence as Fig. 6, but for pig \#2 with the infarcted portion of the free wall shown in Fig. 7. Images were taken $126 \mathrm{~d}$ after infarction. The displacements and velocities remain low and high, respectively, throughout the cardiac cycle. During mid-diastole, a small region of elevated displacements is seen in the infarct, shown by the magenta region in the upper right portion of the images at $412 \mathrm{~ms}$.

\section{B. Intraventricular Septum Infarction}

A comparison of ARFI and SWEI data imaging healthy and infarcted myocardium within the same animal is shown in Fig. 10. The tissue in the infarcted region was thinned, and presented a conduction delay in the B-mode. The ARFI and SWEI M-modes, however, show no apparent change along the lines measured. The healthy tis-
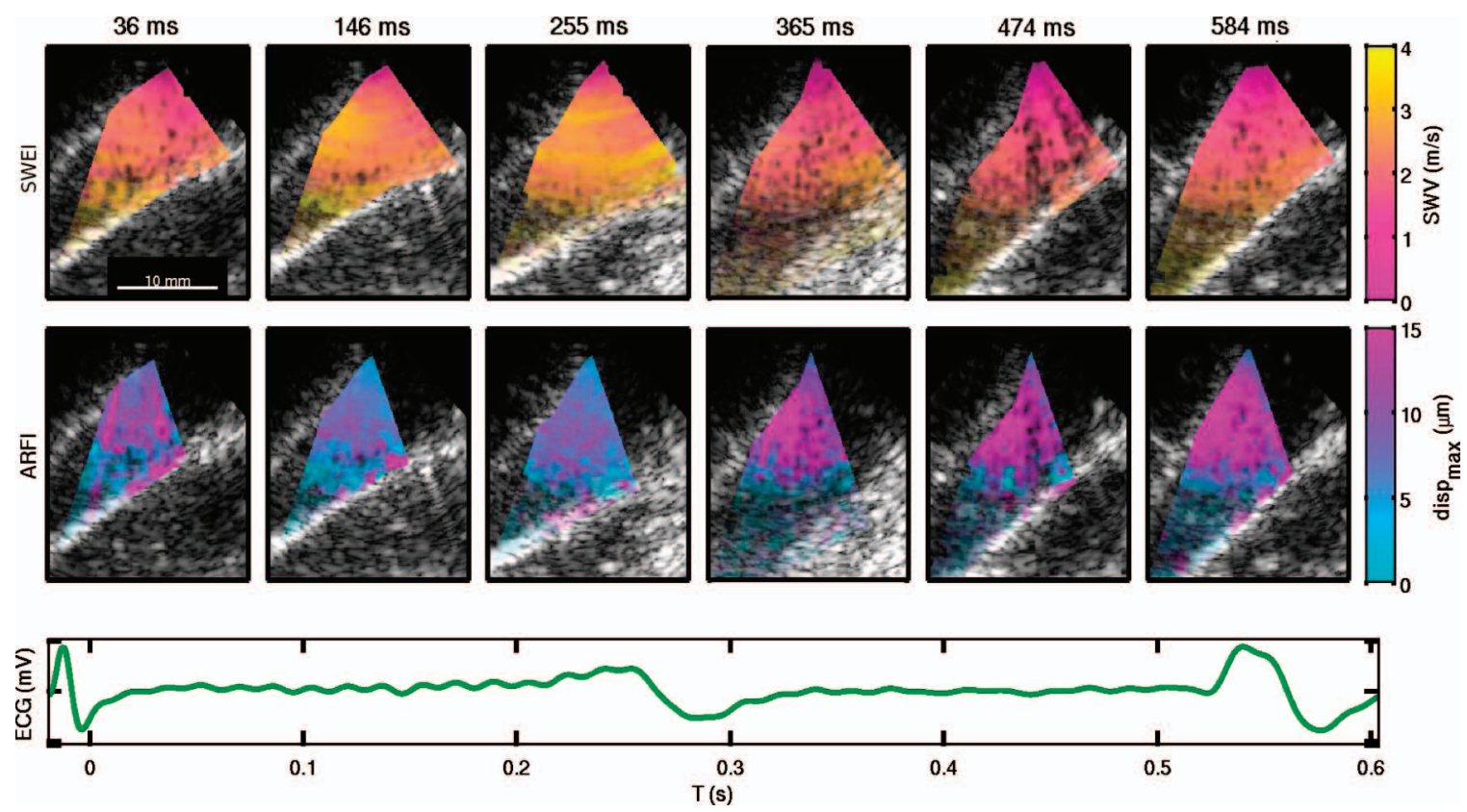

Fig. 6. (top row) SWEI and (middle row) ARFI images of the left ventricular free wall (LVFW) from the control animal at various points in the cardiac cycle, displayed over the corresponding B-mode images, with (bottom row) the associated ECG trace. Similar speeds and displacement trends are seen as in Fig. 5, with general spatial uniformity and temporal contraction. Transparent colored pixels correspond to low cross-correlation coefficients. 

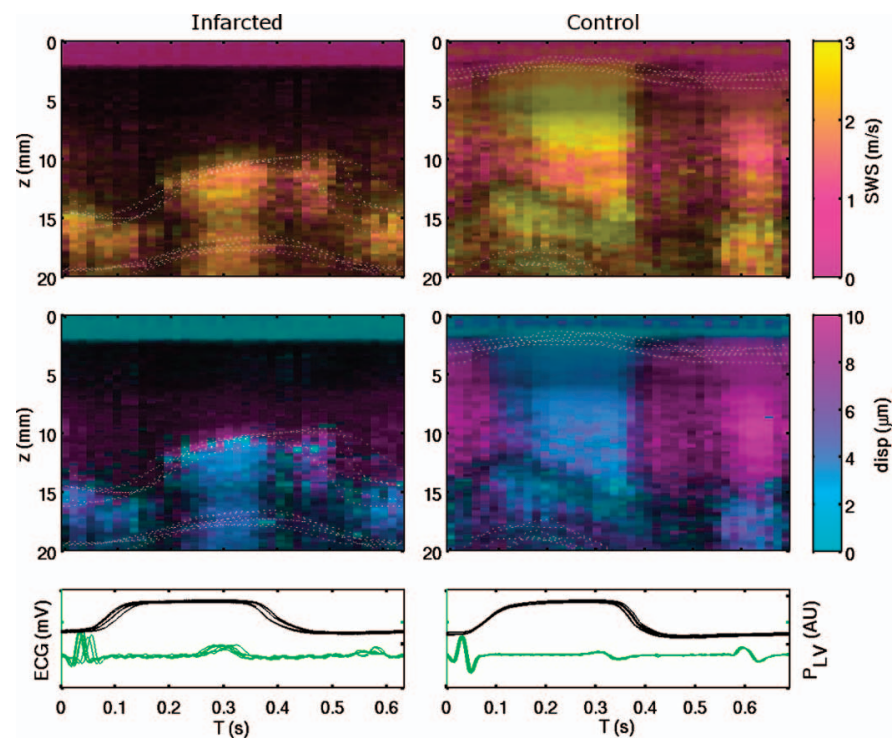

Fig. 7. Multibeat-synthesized (top row) SWEI and (middle row) ARFI M-modes of the left ventricular free wall (LVFW). The left images are taken from an infarcted region of Pig \#2 $126 \mathrm{~d}$ after infarction, and the right images are taken from the control animal at $146 \mathrm{~d}$ after a sham procedure. The bottom row shows the associated ECG and pressure traces. Elevated diastolic shear wave speeds and corresponding lower ARFI displacements are seen in the infarction. Thin, dotted white lines delineate the axial tissue boundaries from all contributing beats, such that overlapping lines indicate repeatability of the axial tissue motion between beats.

sue shows strong shear wave speed variation transmurally through the septum.

Fig. 11 shows the box plots for the views of the IVS shown in Fig. 10, along with the other two repeated ac-

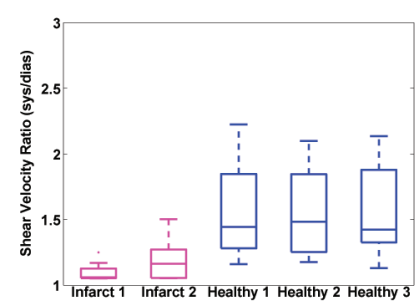

(a)

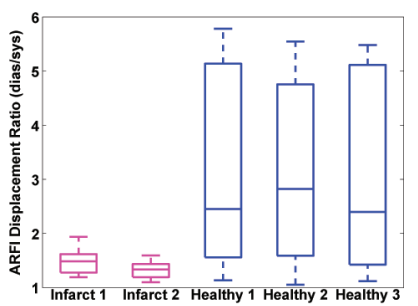

(b)
Fig. 8. Model parameters fit to each of the data sets acquired of the left ventricular free wall (LVFW) view during the final imaging study. Error bars indicate the standard deviation across the LVFW for 10 independent model fits. Data from the control pig, labeled "Healthy," show higher contractility than those from the infarcted pig in both ARFI and SWEI.

quisitions at each view. No statistically significant differences are seen, although the shear wave speed ratios seem to have lower bounds in the spared tissue, and the ARFI ratios seems to be somewhat higher in the spared tissue.

\section{Remodeling}

The ejection fractions for the pigs, from three views, are presented in Table II. All of the animals, including the control, had a moderately decreased ejection fraction 9 to $10 \mathrm{~d}$ after the embolization. Data from the later studies are not available.

The diastolic shear wave speeds, taken from the model fits to all data sets in both the RVFW and the IVS, are shown in Fig. 13. In all but pig \#1, the diastolic shear wave speed increased with significance $p<0.05$ by the final imaging study, including the control animal. In the
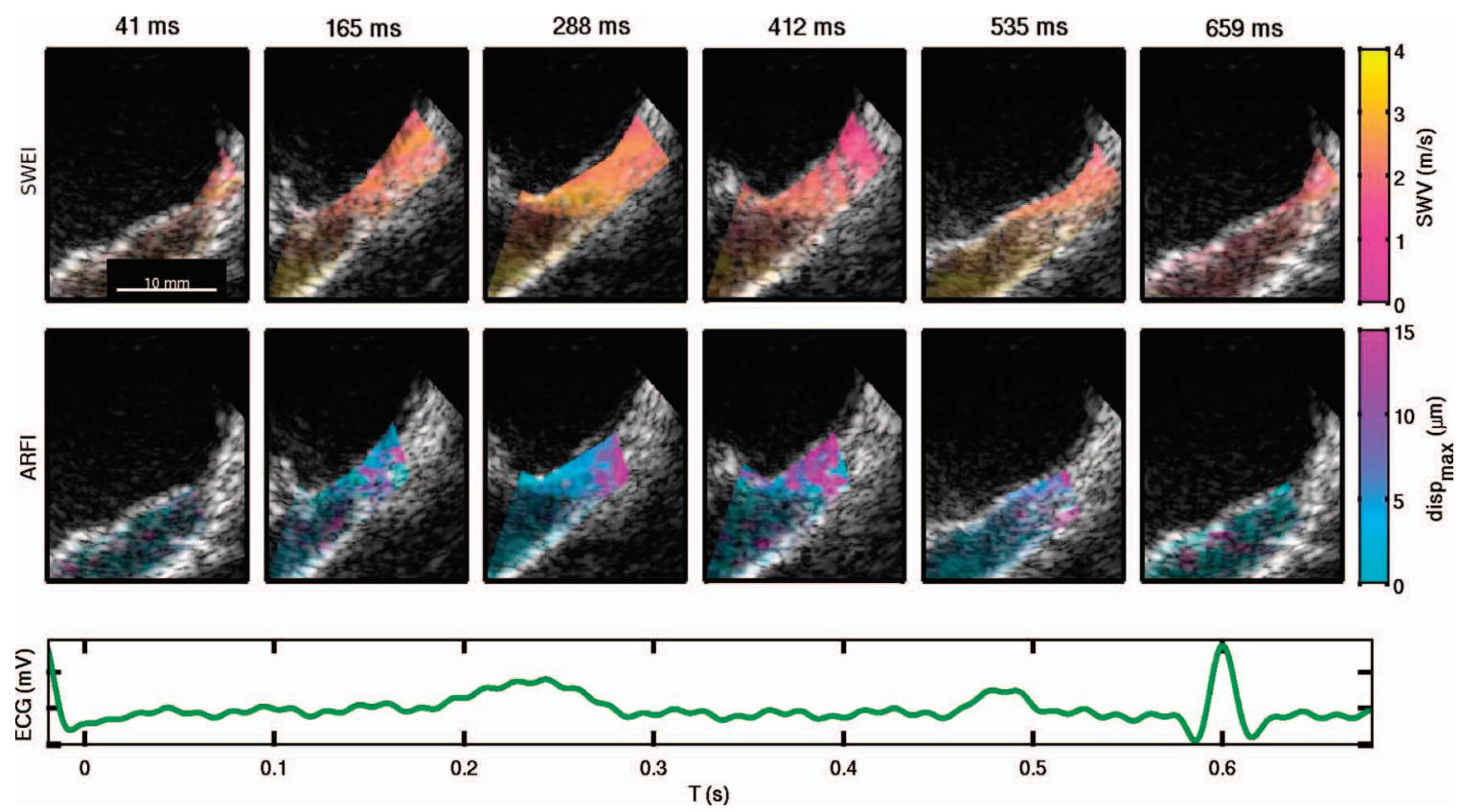

Fig. 9. (top row) SWEI and (middle row) ARFI frames from the synthesized 2-D image sequence of a region of infarcted LVFW, and (bottom row) the corresponding ECG. The infarcted tissue maintains low displacements and high velocities throughout the cardiac cycle, indicating that it is stiff and non-contractile. During mid-diastole $(412 \mathrm{~ms})$, a small region of higher displacements and lower velocities is seen in the upper-right part of the image. 

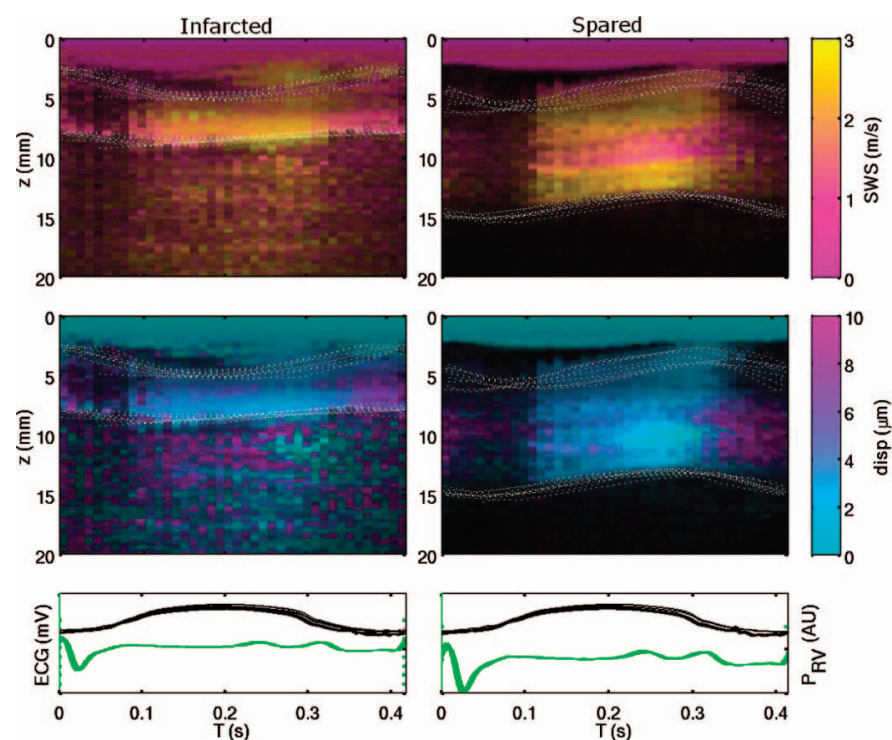

Fig. 10. Multibeat-synthesized M-mode (top row) SWEI and (middle row) ARFI of the intraventricular septum (IVS) for (left) an infarcted region and (right) spared region of the same animal $140 \mathrm{~d}$ after infarction, with (bottom row) traces of ECG and relative pressure. The spared region indicates potential transmural anisotropy, but the infarcted region does not. Though thinned in the infarct, peak contractility appears similar in both tissue samples, and both regions show diastolic compliance.

RVFW, none of the diastolic shear wave velocities were increased at the final imaging study.

Cross-sectionally, comparing all of the views of the IVS from the final imaging study between all of the animals, no differences were seen in diastolic shear wave speeds between pigs. The animals with infarcts had diastolic speeds of $1.289 \pm 0.033,1.354 \pm 0.029$, and $1.366 \pm 0.059$ $\mathrm{m} / \mathrm{s}$, respectively, whereas the control animal had diastolic speeds of $1.420 \pm 0.042 \mathrm{~m} / \mathrm{s}$. If anything, the average diastolic velocities for the control are a bit higher that the infarcted estimates, but grouping the infarcted animal estimates together, and performing a one-way analysis of variance (ANOVA) against the control, the means are not significantly different $(p=0.058)$.

Individual views of non-infarcted septum were also compared across the four pigs. The most common view was of the IVS, and multibeat-synthesized M-modes of the IVS are shown for the four pigs in Fig. 14. Substantial remodeling is not apparent in the ARFI or SWEI data, as clean contractility with similar shear wave speeds is observed. A statistical analysis by one-way ANOVA indicates no difference in the diastolic means between the in-

TABLE II. Ejection Fraction Measurements.

\begin{tabular}{lcc}
\hline $\begin{array}{l}\text { Ejection } \\
\text { fraction }(\%)\end{array}$ & Pre-embolization & $\begin{array}{c}9 \text { to } 10 \mathrm{~d} \\
\text { Post-embolization }\end{array}$ \\
\hline Pig 1 & $58.3 \pm 8.1$ & $54.7 \pm 5.9$ \\
Pig 2 & $52.5 \pm 7.6$ & $51.0 \pm 2.6$ \\
Pig 3 & $51.0 \pm 8.5$ & $40.7 \pm 6.7$ \\
Pig 4 (Control) & $54.3 \pm 3.2$ & $46.0 \pm 3.0$ \\
\hline
\end{tabular}

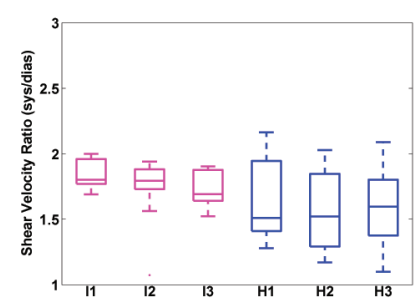

(a)

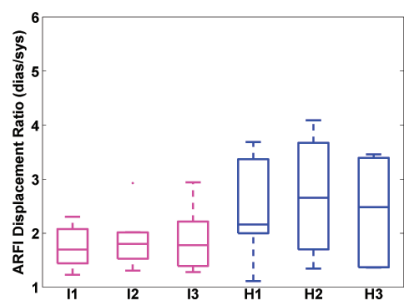

(b)
Fig. 11. Model parameters fit to each of the data sets acquired for the IVS views shown in Fig. 10. Error bars indicate the standard deviation spatially across the septum for 10 independent model fits. SWEI data indicate higher variation in the larger, spared region, but higher ratios in the thinned region, whereas ARFI data indicate higher, though not significantly so, ratios in the spared region.

farcted animals and the healthy (all infarcted $c_{\text {dias }}=1.444$ $\pm 0.059 \mathrm{~m} / \mathrm{s}$, control $\left.c_{\text {dias }}=1.414 \pm 0.102, p=0.801\right)$.

Fig. 15 shows the box plots for the model parameters fit to the data in Fig. 14. Pig Infarct 3 has higher contractility than the control in the ARFI ratio, but not the SWEI ratio, and none of the other diseased pigs showed significant differences in either measurement.

\section{Heterogeneous Infarction}

In one of the animals, views were obtained of a thinned region of apical septum from the LV. The catheter was able to be drawn back, moving the field of view to more basal, spared septum. At approximately $5 \mathrm{~mm}$ intervals, images were taken. A diagram of the approximate imaging locations is shown in Fig. 16. The B-mode shown is from the center of the three locations.

Fig. 17 shows the SWEI and ARFI M-modes recorded for the rightmost line in Fig. 16. Transmural variation of ARFI and SWEI is seen during systole in each beat, with more spatially-uniform values in diastole. These features
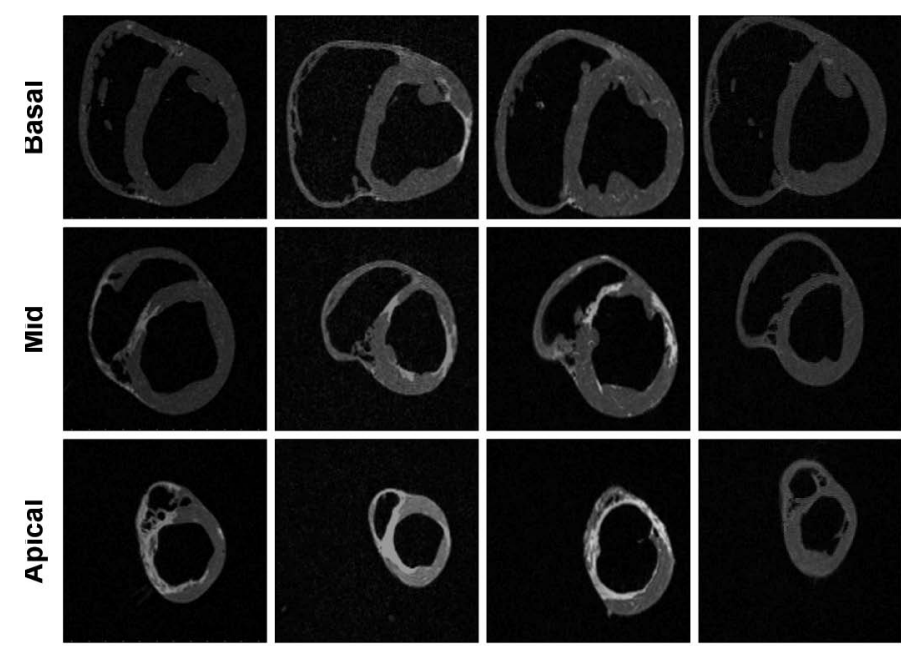

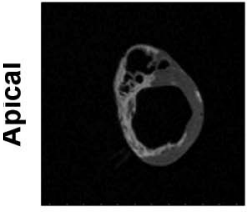

Pig \#1

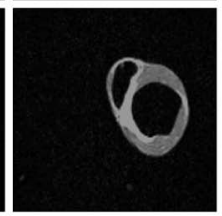

Pig \#2

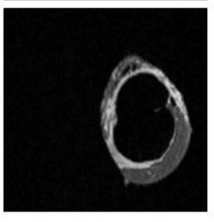

Pig \#3
Pig \#4 (Control)
Fig. 12. Slices of the ex vivo contrast-enhanced MRI volumes. The right ventricles were distended in the fixation process, but infarcts are clearly visible in the IVS of Pigs $1-3$, as well as the left ventricular free wall (LVFW) of pigs 2 and 3. 


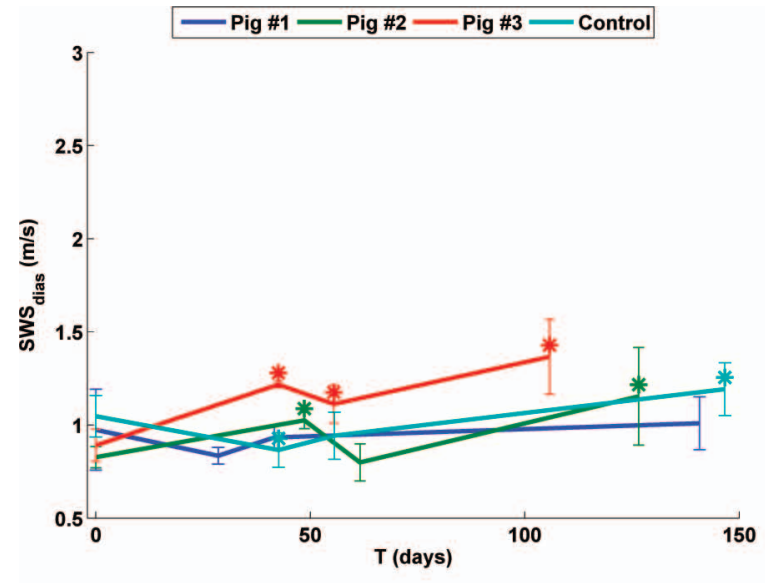

(a)

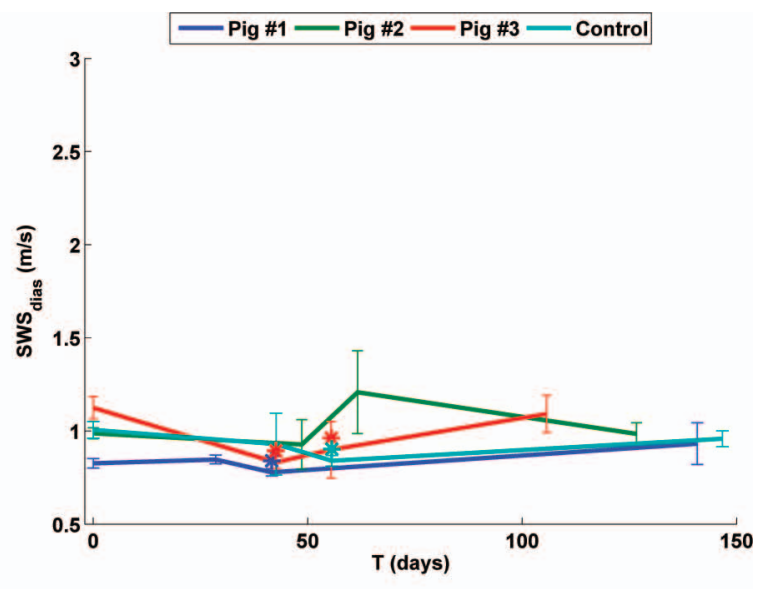

(b)

Fig. 13. Diastolic shear wave speeds in (a) the intraventricular septum (IVS) and (b) right ventricular free wall (RVFW) for the four animals over 150 d. Stastical significance in a Student's two-tailed t-test is shown relative to the first imaging session for each animal, and demarcated with an asterisk.

are present in each beat of the M-mode. Fig. 18 shows the multibeat-synthesized SWEI and ARFI M-modes for the three locations indicated in Fig. 16. The most basal (spared) image is on the left, and the most apical (infarcted) is on the right (the multi-beat synthesis of the data shown in Fig. 17). The spared septum shows uniform contraction transmurally, whereas the infarcted septum is thinned and shows transmural variation in the stiffness and contractility properties. The RV side of the IVS in the right image maintains high displacements and low shear velocities throughout the cardiac cycle.

Fig. 19 shows images from the 2-D synthesized sequence for the view from Fig. 16. The spatio-temporal nonuniformity is highlighted in this view, because the LV and basal regions of the image contract in systole, whereas the apical RV side remains compliant. Visual registration of laterally moving tissue is readily possible with the 2-D field of view.

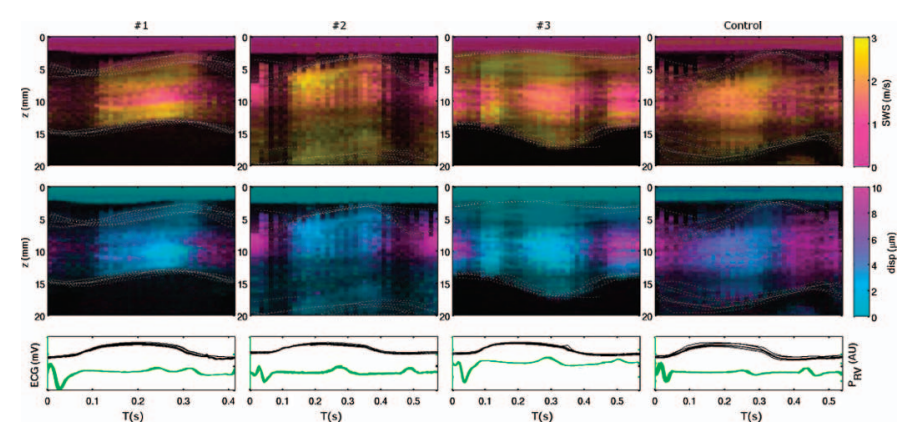

Fig. 14. Multibeat-synthesized M-mode (top row) SWEI and (middle row) ARFI of the intraventricular septum (IVS) for the four pigs, with (bottom row) associated ECG and pressure traces. Images were acquired at the final imaging session for each animal $(140,126$, and $105 \mathrm{~d}$ after infarction, respectively, for Pigs \#1-3, and $146 \mathrm{~d}$ after the sham procedure for the control). No global remodeling is indicated between (left three columns) the spared regions of the IVS in the infarct animals and (right-most column) the healthy tissue of the control.

\section{Discussion}

We were able to use ARF delivered by an ICE transducer to generate and track transient displacements and resultant shear waves through systole and diastole in an animal model. In 122 of the 154 M-mode images, clear trends of higher displacements (increased compliance) during diastole and lower displacements (increased stiffness) during systole were observed. In the remaining 32 images, no cyclic variation was seen - the images had either low displacements within the myocardium through the entire cardiac cycle, or low cross-correlation coefficients indicating motion. In 104 of the 122 M-modes with cyclic ARFI displacements, the matching SWEI images showed clear trends of increased shear wave speeds in systole and decreased speeds in diastole. The high beat-tobeat repeatability facilitated multi-beat synthesis, and the results allowed us to observe interesting spatio-temporal abnormalities in the infarcted myocardium. The ARFI data showed a clean signal indicating at least some systolic contraction in a higher number of M-mode images than the matched SWEI images (79\% versus 68\%) because of the additional step of estimating the transverse

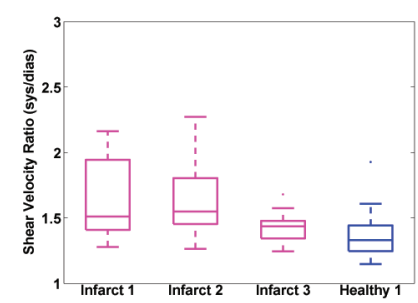

(a)

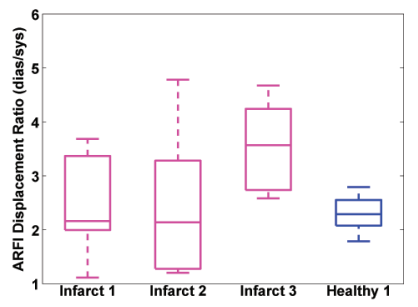

(b)
Fig. 15. Model parameters fit to the data sets acquired from spared septum in the four animals during the final imaging studies. Data from the diseased pigs, labeled "Infarct," show no significant signs of remodeling, compared with the control. The ARFI data show Pig \#3 to have significantly higher ARFI ratios than the control. Error bars indicate standard deviation across the septum among 10 independent model fits. 


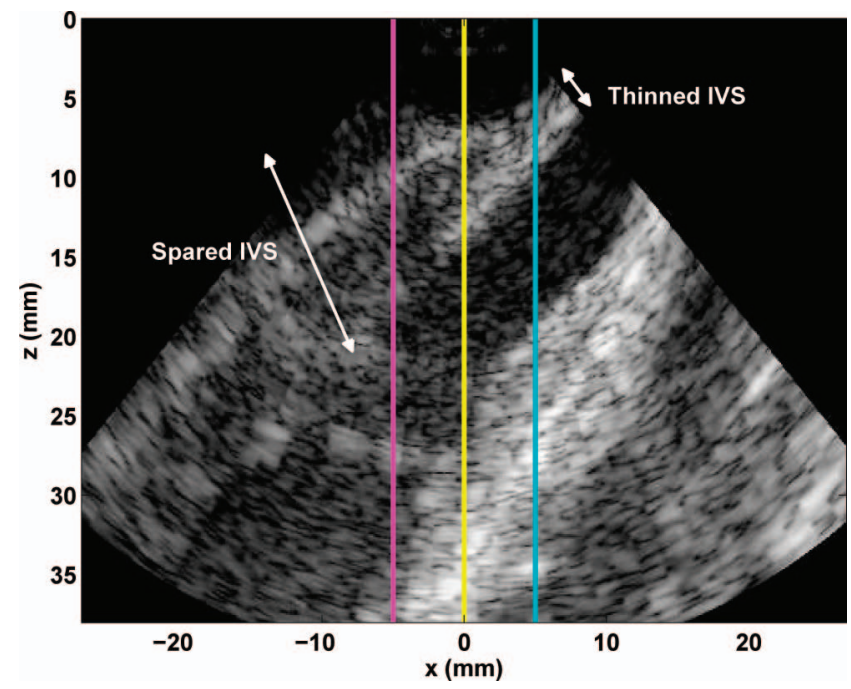

Fig. 16. B-mode image of the intraventricular septum (IVS), with spared septum on the left (basal), and infarcted septum on the right (apical), taken $126 \mathrm{~d}$ after infarction in Pig \#2. The three lines indicate the imaging locations for the M-modes.

wave velocity. ARFI images showed less sensitivity to myocardial fiber orientation than SWEI images, because we were only able to measure the shear wave propagation in a single direction. The combination of both ARFI and SWEI allowed us to separate changes in substrate stiffness from variations in relative fiber orientation, which, without three-dimensional tracking of wave propagation, is not possible with shear wave imaging alone.

\section{A. Left Ventricular Free Wall Infarction}

In the two views of the LVFW, high shear wave speeds and little contraction were seen in the infarcted region
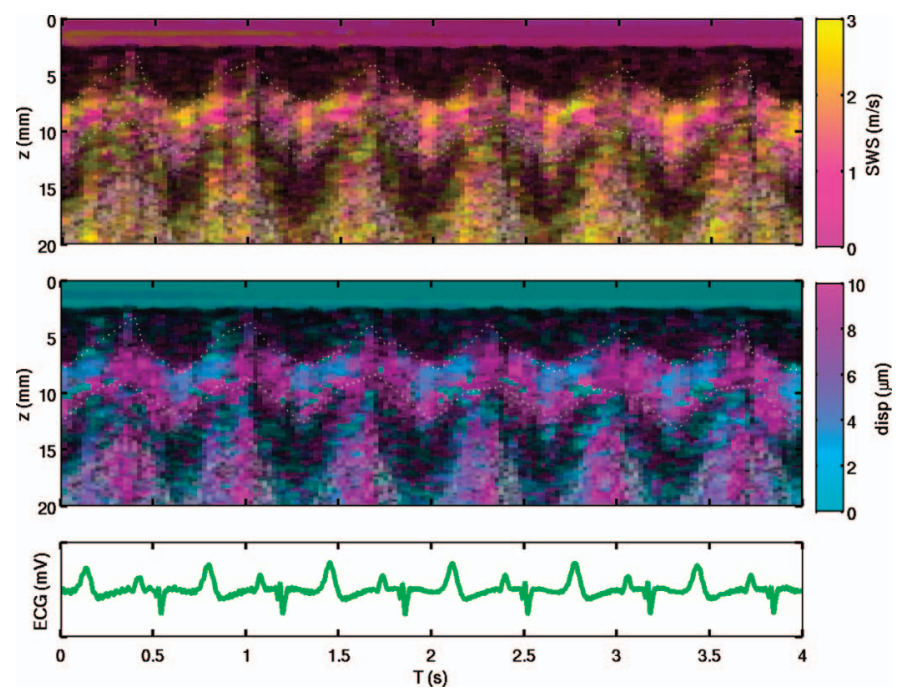

Fig. 17. (top row) SWEI and (middle row) ARFI M-modes of the septum and (bottom row) the corresponding ECG trace, recorded at the rightmost (cyan) line indicated in Fig. 16. Both ARFI and SWEI measurements indicate transmural heterogeneity during systole and axially uniform estimates in diastole, $126 \mathrm{~d}$ after infarction. These spatio-temporal trends are visible in each beat.
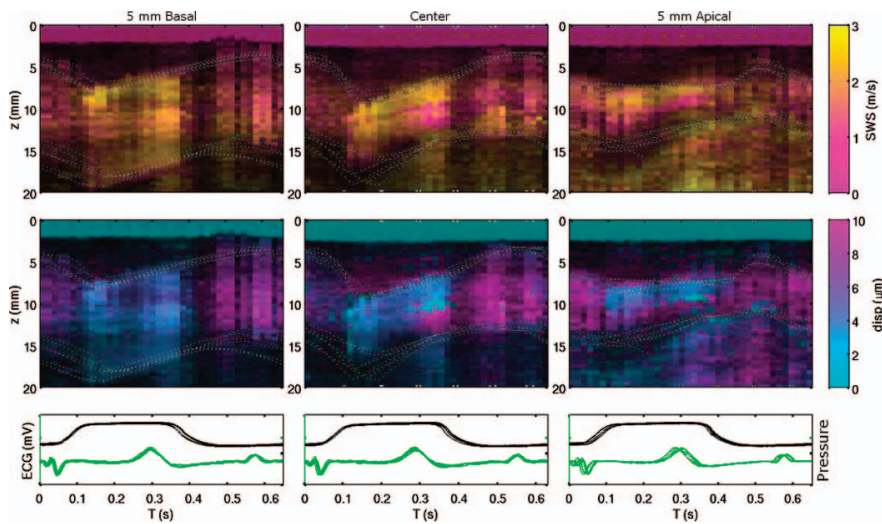

Fig. 18. Multibeat-synthesized (top row) SWEI and (middle row) ARFI M-modes of the septum, at the locations indicated in Fig. 16, with (bottom row) corresponding ECG and pressure traces. The apical septum is thinned and shows transmural variation in systolic stiffness in both ARFI and SWEI measurements for the center and apical views (middle and right columns) $126 \mathrm{~d}$ after infarction.

compared with the healthy tissue. The relatively higher variance of the ARFI estimates for the healthy tissue (Fig. 8 ) is due to depth of field and attenuation effects over the much larger axial thickness of the free wall, which extends beyond $20 \mathrm{~mm}$. Fig. 5 shows that the axial and temporal trends through the LVFW and across the heart cycle are shown with high beat-to-beat repeatability. Looking at the upper end of each box for ARFI or SWEI in Fig. 8 indicates large differences between the infarcted and control tissues in terms of the spatial peak contractility. The 2-D image sequences (Figs. 6 and 9) show the relative spatial homogeneity of each of the tissue regions. The 2-D SWEI images have low resolution because of the averaging of overlapping $16^{\circ}$ ROIs but, with the higher-resolution ARFI images, do provide insight into the azimuthal distribution of stiffness. Interestingly, in the healthy tissue, a gradient is visible with depth during diastole, potentially indicating somewhat increased stiffness toward the epicardium. These low displacements and elevated velocities at depth during diastole are seen in the deep parts of Fig. 7 as well. This may indicate that the epicardial part of the myocardium is more rigid than the endocardium, but effects of boundary conditions and ARF penetration depth obfuscate a stronger conclusion.

\section{B. Intraventricular Septum Infarction}

The views of the IVS (Fig. 10) show a primarily negative result for distinguishing what appeared to be infarcted tissue from normal tissue within the same heart by ARFI and SWEI. The shear wave speeds and ARFI displacements in the thinned myocardium seem to follow the same contraction and relaxation of the spared region. Several factors complicate this conclusion, however. First, the IVS displays transmural anisotropy in the shear wave speed images of the spared region, but not in the infarcted region. The band in the middle of the spared septum shows low shear wave speeds and low displacements during systole, 

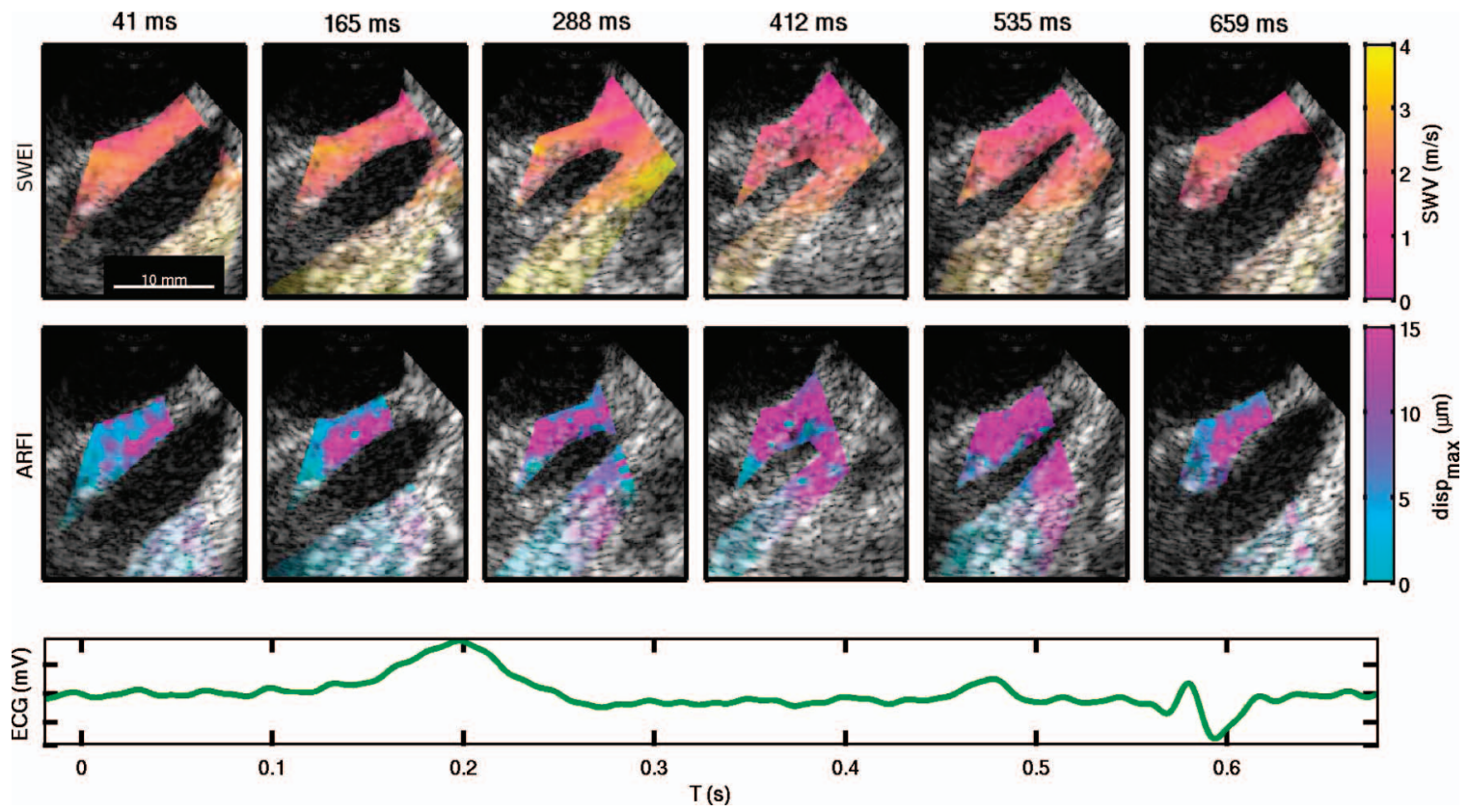

Fig. 19. (top row) SWEI and (middle row) ARFI frames from the 2-D image sequence of a heterogeneously-infarcted region (Fig. 16), and (bottom row) the corresponding ECG trace. In the ARFI images, the right ventricle (RV) side of the intraventricular septum (IVS) near the apex does not contract during systole, showing elevated displacements, whereas the left ventricle (LV) side of the IVS contracts and relaxes normally.

but no such region exists in the presumed infarct. This causes the average systolic shear wave speed to be lower, and the standard deviation to be higher, in the spared region, as seen in Fig. 11(a). The ARFI ratios, on the other hand, appear somewhat higher in the spared septum, though statistical significance is not achieved. Neither of these change the fact, however, that the thinned region of myocardium is compliant during diastole, with shear wave speed near $1 \mathrm{~m} / \mathrm{s}$. We hypothesized that this could be stunned or hibernating myocardium, which would be dysfunctional without being fibrotic. Although the tissue may not actively contract itself, the contraction of adjacent, healthy tissue may stretch this tissue, creating the systolic stiffness. However, neither the ex vivo MRI (Fig. 12), nor the histological staining (not shown) indicated that pig \#1 had a thinned region of its septum that was not infarcted. The existence of this apparent soft infarction warrants further study to determine whether it is the result of an imaging artifact or a real pathology.

\section{Remodeling}

Over the course of the study, we observed a small stiffening of the IVS during diastole in two of the three infarcted pigs as well as the control, and no significant change in RVFW diastolic stiffness, leading us to believe that we either did not achieve, or were not able to detect diastolic heart failure in these animals. The ejection fractions (Table II) did not differentiate the infarcted animals from the control at 9 to $10 \mathrm{~d}$ post-embolization, although in the later studies, regional akinesis of the infarct areas was observed, and expected to have decreased the ejection fractions below normal, although those data are un- available. Additionally, cross-sectionally comparing all of the diastolic shear wave speeds in regions of spared IVS during the final imaging session, no signs of elevated diastolic stiffness were present in the SWEI data for the infarcted animals. Comparing similar views of the septum (Fig. 14), we see variation, but no significant differences between the spared regions of the infarcted pigs and those of the control. The average diastolic velocities were nearly identical $(1.414 \mathrm{~m} / \mathrm{s}$ in the control, $1.444 \mathrm{~m} / \mathrm{s}$ in the infarcted animals, $p=0.801)$. Fig. 14 shows the M-mode images taken of the IVS, and we can see that the exact imaging plane varies between the views. The variation introduced by different imaging angles and targets indicates the importance of obtaining measurements of the same part of the tissue, at the same angle, for performing matched comparisons. Perhaps the strongest conclusion to be drawn from these data is that the heart is not mechanically homogeneous and that substantial regional variation exists even in healthy hearts. To make the truest comparison between diseased tissue and healthy, either the imaging planes must be closely matched, or a map of regional properties must be created that can be aligned and registered between subjects.

\section{Heterogeneous Infarction}

In Figs. 18 and 19, ARFI and SWEI agree on the decreased systolic stiffness of the RV side of the IVS near the apex, so the variation cannot be attributed simply to anisotropy. This result, like the other IVS result, was surprising, because it was expected that the thinned myocardium would be stiff and noncompliant throughout the cardiac cycle. Ex vivo contrast-enhanced MRI indicated 
the apical septum to be infarcted, but aligning the specific ultrasound viewing angles to the MRI data was not possible because of deformations introduced in fixing the heart for scanning. It is possible that the ultrasound imaging plane intersected infarcted, viable, or a mix of both types of tissue at different stages of the cardiac cycle. This infarcted region of the IVS may have been subjected to passive tension as the surrounding tissue contracted, such that the increase in stiffness on the LV side during systole was due to stretching rather than muscular contraction. Another confounding factor was substantial lateral motion of the septum in these images. The tissue moved back and forth underneath the ROI, and in the apical image, the ROI approached the apex itself at the peak lateral motion. Looking at the 2-D images, however (Fig. 19), clear elastic distinction between the RV and LV sides of the septum was observed during systole in the ARFI images, and the moving septum is readily followed through the frames.

\section{E. Limitations}

To limit the anesthesia time of the animals, the data acquired were processed offline after each experiment. Single 2-D diastolic ARFI-only images were typically acquired before each set of M-mode and 2-D ARFI and SWEI images to determine the appropriate focal configuration to use, but feedback on the quality of the acquired data was limited during the experiments. Images were typically acquired in whatever views were provided by the tissue within $2 \mathrm{~cm}$ of the transducer face with limited perceived motion, but the porcine cardiac anatomy created challenges in catheter positioning, and in some cases, the only available views of the septum or free wall had a large amount of motion.

Lateral and elevational wall motion, particularly during contraction and active relaxation, created decorrelation in the tracking data, which reduced the confidence of the displacement estimates and subsequently-calculated velocities. In the images shown, the saturation of each pixel is tied to the cross-correlation coefficients of the contributing excitation-tracking ensemble, and low cross-correlation coefficients exponentially de-weight the saturation, showing more of the underlying B-mode, as shown in Fig. 17. This occurs in blood and in regions of high motion. The tissue is outlined with small dotted white lines in the M-modes to show the tissue boundaries, but in the 2-D images, the blood signal was manually masked out, rather than just using the correlation coefficients. When making multi-beat synthesized M-modes, the multi-beat averaging was weighted by the correlation coefficients to reduce the effect of outliers, but the motion from beat to beat is so repeatable that decorrelated pixels were often averaged with the also-decorrelated pixels from the following beats, as in Fig. 18. Real-time guidance of ARFI and SWEI will aid future studies in imaging plane alignment, particularly with respect to fiber orientation. Additionally, as the heart beats, the angle between the ARF excitation and the tissue may change with cardiac motion. In the Mmode images, this may mean that a different part of the tissue is imaged in systole than in diastole. Although the multibeat-synthesis 2-D image sequences address this, some M-mode results could be subject to potential artifacts caused by the lateral and elevational heterogeneity of the tissue around the focus.

The effect of fiber orientation was more of a confounding factor on the shear wave speed data than was initially expected. Although ARF excitations occur mostly perpendicular to fibers, shear wave propagation is tracked by a linear phased array in a specific dimension, which can be parallel or perpendicular to the fiber orientation. ARFI was therefore less affected than SWEI, and in cases where the shear wave speeds varied without a corresponding change in the ARFI, we expect to be imaging fiber orientation variation rather than substrate stiffness changes. Future studies of shear wave speeds and myocardium will have to control for relative fiber angle closely, or else move toward tracking the wave propagation in 3-D, which would allow the anisotropic shear wave propagation to be imaged in all directions.

Beyond anisotropy, frequency dispersion affects the generalization of the shear wave speed estimates. In some views, the myocardium is less than $5 \mathrm{~mm}$ thick, and a shear wave model may not be appropriate because the tissue may be excited in an antisymmetric Lamb wave mode. Nenadic et al. [38] describe an approach for modeling such dispersion. In addition to boundary-condition-induced dispersion, myocardium is also expected to exhibit a degree of dispersion associated with its viscoelastic properties. How these properties provide different information than the basic group velocity approach used here will also need to be explored in future work.

Perhaps the greatest limiting factor, however, is the limited range of imaging. Because the AcuNav is such a small probe, it was difficult to generate and track shear waves at depth. Although images were acquired with up to a $45^{\circ}$ field of view down to $4 \mathrm{~cm}$, images with excitation foci beyond $15 \mathrm{~mm}$ were less successful at generated distinct shear waves than those which used transmit Fnumbers less than 2 . This limited the usable field of view to a small region, which in turn limited the amount of the heart that could be characterized in any single image. Some views did show spatial variation within the small window (Fig. 19, for example), but we predict that the capabilities of the system would greatly benefit from an increase in the usable field of view provided by stronger, more focused excitations. A larger transducer, an elevational focus, or longer excitation pulses may provide options in the future for accomplishing this. A wider viewing angle for tracking the wave may also provide better separation of the tissue motion from the ARF-induced motion and eliminate the need for multiple excitations per SWEI estimate altogether. This may be achieved through the higher receive bandwidth of newer hardware, either with parallel beamforming of many beams or through coherent plane wave compounding [39]. 


\section{CONCLUSion}

This work explored the possibility of using SWEI and ARFI M-mode and synthesized 2-D measurements to characterize infarcted myocardium. Spatio-temporal abnormalities were observed in the SWEI and ARFI data that corresponded to regions of myocardial infarct, and these abnormalities were repeatedly imaged across several beats. Variability between imaging planes resulting from anisotropy, viewing angle, and tissue motion confounds generalization of these results, and highlights the need for tightly controlling for catheter position. Some abnormal regions showed increased systolic compliance, whereas others showed decreased diastolic compliance. These results indicate the potential for ICE ARFI and SWEI techniques to be used to characterize diseased myocardial stiffness directly, but also highlight the complexity of performing elasticity imaging in beating myocardium.

\section{ACKNowledgments}

The authors thank E. Dixon-Tulloch for assistance on the porcine studies, Siemens Medical Systems for technical support, Synecor Labs for their work on the animal experiments, Dr. H. Kim's group for performing the ex vivo MRIs, S. Eyerly and B. Potter for slicing and staining the hearts, and all of the graduate students and research professors in Dr. Trahey's and Dr. Nightingale's labs for ARFI sequencing ideas and advice.

\section{REFERENCES}

[1] D. K. Bogen, S. A. Rabinowitz, A. Needleman, T. A. McMahon, and W. H. Abelmann, "An analysis of the mechanical disadvantage of myocardial infarction in the canine left ventricle," Circ. Res., vol. 47, no. 5, pp. 728-741, 1980.

[2] R. G. McKay, M. A. Pfeffer, R. C. Pasternak, J. E. Markis, P. C. Come, S. Nakao, J. D. Alderman, J. J. Ferguson, R. D. Safian, and W. Grossman, "Left ventricular remodeling after myocardial infarction: A corollary to infarct expansion," Circulation, vol. 74 , no. 4 , pp. 693-702, 1986.

[3] P. Uusimaa, J. Risteli, M. Niemelä, J. Lumme, M. Ikäheimo, A. Jounela, and K. Peuhkurinen, "Collagen scar formation after acute myocardial infarction," Circulation, vol. 96, no. 8, pp. 2565-2572, 1997.

[4] R. J. Kim, D. S. Fieno, T. B. Parrish, K. Harris, E.-L. Chen, O. Simonetti, J. Bundy, J. P. Finn, F. J. Klocke, and R. M. Judd, "Relationship of MRI delayed contrast enhancement to irreversible injury, infarct age, and contractile function," Circulation, vol. 100, no. 19 , pp. 1992-2002, 1999.

[5] R. J. Kim, E. Wu, A. Rafael, E.-L. Chen, M. A. Parker, O. Simonetti, F. J. Klocke, R. O. Bonow, and R. M. Judd, "The use of contrast-enhanced magnetic resonance imaging to identify reversible myocardial dysfunction," N. Engl. J. Med., vol. 343, no. 20, pp. 1445-1453, 2000.

[6] J. A. C. Lima, R. M. Judd, A. Bazille, S. P. Schulman, E. Atalar, and E. A. Zerhouni, "Regional heterogeneity of human myocardial infarcts demonstrated by contrast-enhanced MRI," Circulation, vol. 92, no. 5, pp. 1117-1125, 1995.

[7] P. G. Masci and J. Bogaert, "Post myocardial infarction of the left ventricle: The course ahead seen by cardiac MRI," Cardiovasc. Diagn. Ther., vol. 2, no. 2, pp. 113-127, 2012.
[8] I. Matsunari, J. Taki, K. Nakajima, N. Tonami, and K. Hisada, "Myocardial viability assessment using nuclear imaging," Ann. Nucl. Med., vol. 17, no. 3, pp. 169-179, 2003.

[9] A. H. Mahnken, R. Koos, M. Katoh, J. E. Wildberger, E. Spuentrup, A. Buecker, R. W. Günther, and H. P. Kühl, "Assessment of myocardial viability in reperfused acute myocardial infarction using 16-slice computed tomography in comparison to magnetic resonance imaging," J. Am. Coll. Cardiol., vol. 45, no. 12, pp. 2042-2047, 2005.

[10] E. W. M. Gruseels, J. W. Deckers, A. W. Hoes, J. A. M. Hartman, E. D. Van Does, E. V. Loenen, and M. L. Simoons, "Pre-hospital triage of patients with suspected myocardial infarction," Eur. Heart J., vol. 16, no. 3, pp. 325-332, 1995.

[11] A. M. Hauser, V. Gangadharan, R. G. Ramos, S. Gordon, G. C. Timmis, and P. Dudlets, "Sequence of mechanical, electrocardiographic and clinical effects of repeated coronary artery occlusion in human beings: Echocardiographic observations during coronary angioplasty," J. Am. Coll. Cardiol., vol. 5, no. 2, pt. 1, pp. 193-197, 1985.

[12] J. J. Heger, A. E. Weyman, L. S. Wann, J. C. Dillon, and H. Feigenbaum, "Cross-sectional echocardiography in acute myocardial infarction: Detection and localization of regional left ventricular asynergy," Circulation, vol. 60, no. 3, pp. 531-538, 1979.

[13] R. Bolli, "Mechanism of myocardial "stunning"," Circulation, vol. 82, no. 3, pp. $723-738,1990$.

[14] E. Braunwald and R. A. Kloner, "The stunned myocardium: Prolonged, postischemic ventricular dysfunction," Circulation, vol. 66 , no. 6 , pp. 1146-1149, 1982.

[15] J. Hare, "The etiologic basis of congestive heart failure," in Atlas of Heart Failure, 5th ed., W. Colucci, Ed., New York, NY: Springer, 2007, ch. 3, pp. 29-56.

[16] F. Haas, C. Haehnel, W. Picker, S. Nekolla, S. Martinoff, H. Meisner, and M. Schwaiger, "Preoperative positron emission tomographic viability assessment and perioperative and postoperative risk in patients with advanced ischemic heart disease," J. Am. Coll. Cardiol., vol. 30, no. 7, pp. 1693-1700, 1997.

[17] P. R. Pagley, G. A. Beller, D. D. Watson, L. W. Gimple, and M. Ragosta, "Improved outcome after coronary bypass surgery in patients with ischemic cardiomyopathy and residual myocardial viability," Circulation, vol. 96, no. 3, pp. 793-800, 1997.

[18] R. Bouchard, S. Hsu, P. Wolf, and G. Trahey, "In vivo cardiac, acoustic-radiation-force-driven, shear wave velocimetry," Ultrason. Imaging, vol. 31, no. 3, pp. 201-213, Jul. 2009.

[19] R. Bouchard, S. Hsu, M. Palmeri, N. Rouze, K. Nightingale, and G. Trahey, "Acoustic radiation force-driven assessment of myocardial elasticity using the displacement ratio rate (DRR) method," Ultrasound Med. Biol., vol. 37, no. 7, pp. 1087-1100, Jul. 2011.

[20] M. Couade, M. Pernot, E. Messas, A. Bel, M. Ba, A. Hagege, M. Fink, and M. Tanter, "In vivo quantitative mapping of myocardial stiffening and transmural anisotropy during the cardiac cycle," IEEE Trans. Med. Imaging, vol. 30, no. 2, pp. 295-305, Feb. 2011.

[21] S. Hsu, R. Bouchard, D. Dumont, P. Wolf, and G. Trahey, "In vivo assessment of myocardial stiffness with acoustic radiation force impulse imaging," Ultrasound Med. Biol., vol. 33, no. 11, pp. $1706-$ 1719, Nov. 2007.

[22] H. Kanai, "Propagation of spontaneously actuated pulsive vibration in human heart wall and in vivo vescoelasticity estimation," IEEE Trans Ultrason. Ferroelectr. Freq. Control, vol. 52, no. 11, pp. 1931-1942, Nov. 2005.

[23] M. Pernot, M. Couade, P. Mateo, B. Crozatier, R. Fischmeister, and M. Tanter, "Real-time assessment of myocardial contractility using shear wave imaging," J. Am. Coll. Cardiol., vol. 58, no. 1, pp. 65-72, Jun. 2011.

[24] C. Pislaru, M. Urban, I. Nenadic, and J. Greenleaf, "Shearwave dispersion ultrasound vibrometry applied to in vivo myocardium," in 31st Annu. Int. Conf. IEEE Engineering in Medicine and Biology Society, 2009, pp. 2891-2894.

[25] E. Chu, A. Fitzpatrick, M. Chin, K. Sudhir, P. Yock, and M. Lesh, "Radiofrequency catheter ablation guided by intracardiac echocardiography," Circulation, vol. 89, no. 3, pp. 1301-1305, 1994.

[26] E. G. Daoud, S. J. Kalbfletsch, and J. D. Hummel, "Intracardiac echocardiography to guide transseptal left heart catheterization for radio-frequency catheter ablation," J. Cardiovasc. Electrophysiol., vol. 10, no. 3, pp. 358-363, 1999.

[27] N. F. Marrouche, D. O. Martin, O. Wazni, A. M. Gillinov, A. Klein, M. Bhargava, E. Saad, D. Bash, H. Yamada, W. Jaber, R. Schweikert, P. Tchou, A. Abdul-Karim, W. Saliba, and A. Natale, "Phasedarray intracardiac echocardiography monitoring during pulmonary 
vein isolation in patients with atrial fibrillation," Circulation, vol. 107, no. 21, pp. 2710-2716, 2003.

[28] M. Mullen, B. Dias, F. Walker, S. Siu, L. Benson, and P. McLaughlin, "Intracardiac echocardiography guided device closure of atrial septal defects," J. Am. Coll. Cardiol., vol. 41, no. 2, pp. 285-292, Jan. 2003.

[29] S. Vaina, J. Ligthart, M. Vijayakumar, F. T. Cate, M. Witsenburg, L. Jordaens, G. Sianos, A. Thornton, M. Scholten, P. de Jaegere, and P. Serruys, "Intracardiac echocardiography during interventional procedures," EuroIntervention, vol. 1, no. 4, pp. 454-464, February. 2006.

[30] P. Hollender, P. Wolf, and G. Trahey, "Intracardiac shear wave velocimetry using acoustic radiation force (ARF) excitations: In vivo results," presented at the IEEE Int. Ultrasonics Symp., Orlando, FL, Oct. 18-21, 2011.

[31] S. Hsu, B. Fahey, D. Dumont, and G. Trahey, "Acoustic radiation force impulse imaging with an intra-cardiac probe," in Proc. SPIE, 2005, p. 196.

[32] M. L. Palmeri and K. R. Nightingale, "On the thermal effects associated with radiation force imaging of soft tissue," IEEE Trans. Ultrason. Ferroelectr. Freq. Control, vol. 51, no. 5, pp. 551-565, 2004.

[33] C. Kasai, K. Namekawa, A. Koyano, and R. Omoto, "Real-time twodimensional blood flow imaging using autocorrelation technique," IEEE Trans. Sonics Ultrason., vol. 32, no. 3, pp. 458-463, 1985.

[34] D. Giannantonio, D. Dumont, G. Trahey, and B. Byram, "Comparison of physiological motion filters for in vivo cardiac ARFI," Ultrason. Imaging, vol. 33, no. 2, pp. 89-108, 2011.

[35] M. Palmeri, M. Wang, J. Dahl, K. Frinkley, and K. Nightingale, "Quantifying hepatic shear modulus in vivo using acoustic radiation force," Ultrasound Med. Biol., vol. 34, no. 4, pp. 546-558, 2008.

[36] A. Sarvazyan, O. Rudenko, S. Swanson, J. Fowlkes, and S. Emelianov, "Shear wave elasticity imaging; A new ultrasonic technology of medical diagnostics," Ultrasound Med. Biol., vol. 24, no. 9, pp. 1419-1436, Dec. 1998.

[37] S. Hsu, B. Fahey, D. Dumont, P. Wolf, and G. Trahey, "Challenges and implementation of radiation-force imaging with an intracardiac ultrasound transducer," IEEE Trans. Ultrason. Ferroelectr. Freq. Control, vol. 54, no. 5, pp. 996-1009, May 2007.

[38] I. Nenadic, M. Urban, S. Mitchell, and J. Greenleaf, "Lamb wave dispersions ultrasound vibrometry (lduv) method for quantifying mechanical properties of viscoelastic solids," Phys. Med. Biol., vol. 56, no. 7, pp. 2245-2264, 2011.

[39] G. Montaldo, M. Tanter, J. Bercoff, N. Benech, and M. Fink, "Coherent plane-wave compounding for very high frame rate ultrasonography and transient elastography," IEEE Trans. Ultrason. Ferroelectr. Freq. Control, vol. 56, no. 3, pp. 489-506, Mar. 2009.

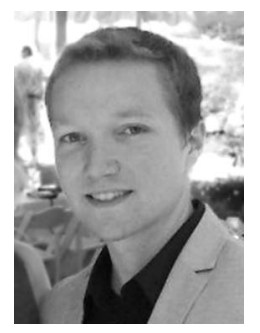

Peter Hollender (S'12) was born in Iowa City, IA, in 1987. He earned his B.S.E. degree in biomedical engineering and electrical and computer engineering from the Pratt School of Engineering, Duke University, Durham, NC, in 2009, and is currently a Ph.D. candidate in biomedical engineering at Duke University. He has held internships at NASA, RoviSys, and Siemens Healthcare. Mr. Hollender has served as the student representative for ultrasonics to the IEEE Ultrasonics, Ferroelectrics, and Frequency Control Society since 2012, and as a National Collegiate Inventors and Innovators Alliance student ambassador for innovation and entrepreneurship since 2013. His research interests include novel methods for cardiac elasticity imaging, guidance of surgical procedures with ultrasound, and intracardiac echocardiography.

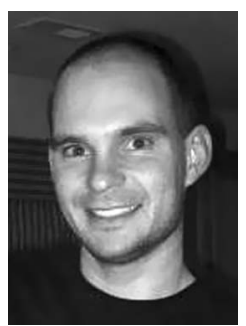

David P. Bradway (S'02) received his B.S. degree in electrical and computer engineering from the Ohio State University, Columbus, OH, in 2005 and his $\mathrm{Ph} . \mathrm{D}$. degree in biomedical engineering at Duke University, Durham, NC, in 2013. He has completed research internships at the Cleveland Clinic Foundation, Volcano Corporation, and Siemens Healthcare. His research interests include acoustic radiation force imaging methods and ultrasonic cardiac elasticity imaging.

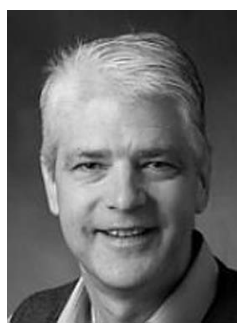

Patrick Wolf was born in Altoona, PA, in 1956. He received his undergraduate degree in electrical engineering from the Pennsylvania State University in 1978. He received an M.S. degree in bioengineering from Penn State in 1986 and a Ph.D. degree in biomedical engineering from Duke University in 1992. Dr. Wolf worked as a Research Engineer at the Milton S. Hershey Medical Center from 1978 to 1983 , when he moved to a research position and eventually a postdoctoral position in the laboratory of Raymond E. Ideker studying fibrillation and defibrillation. In 1994, he joined the faculty of the Department of Biomedical Engineering at Duke University. Dr. Wolf is a member of IEEE and the Biomedical Engineering Society (BMES). His current research interests include ablation of cardiac arrhythmias, cardiac and neural electrophysiology, and medical instrumentation related to these fields.

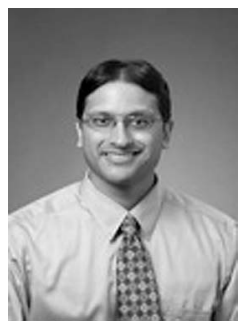

Robi Goswami was born in Denver, CO, in December of 1978. He received B.S. and M.S. degrees in electrical engineering from Stanford University. He subsequently received an M.D. degree from the Duke University School of Medicine, Durham, NC. He was employed as a house staff in Internal Medicine at Duke University Hospital from 2006 to 2009. He is currently completing a Cardiology fellowship at Duke University Hospital. Additionally, he has been pursuing research studies under the mentorship of Gregg Trahey in the Biomedical Engineering Department at Duke University. Dr. Goswami is a member of the American College of Cardiology. He has received the Barton Haynes award for resident research during his training.

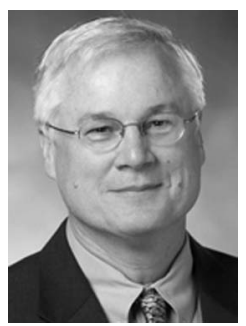

Gregg E. Trahey (S'83-M'85) received the B.G.S. and M.S. degrees from the University of Michigan, Ann Arbor, MI, in 1975 and 1979, respectively. He received the Ph.D. degree in biomedical engineering in 1985 from Duke University. He served in the Peace Corps from 1975 to 1978 and was a project engineer at the Emergency Care Research Institute in Plymouth Meeting, PA, from 1980 to 1982 . He is currently a Professor with the Department of Biomedical Engineering at Duke University and holds a secondary appointment with the Department of Radiology at the Duke University Medical Center. His current research interests include adaptive phase correction and beamforming, and acoustic radiation force imaging methods. 